
\title{
28 Research Suare \\ Ten-year trend of mortality among patients hospitalized with Malaria in Ghana,
}

Keziah Malm ( $\nabla$ kezmalm@yahoo.com )

national malaria control program

Nana Yaw Peprah

Ghana Health Service

Wahjib Mohammed

Ghana Health Service

Nyonuku A. Baddoo

Ghana Health Service

Marijanatu Abdulai

Ghana Health Service

Zeleke Alebachew

Ghana Health Service

Frank Adae Bonsu

Ghana Health Service

Stephen Ayisi Addo

Ghana Health Service

Mark Saalfeld

Global Fund

Donna Lee

Global Fund

Nibretie Workneh

Global Fund

Constance Bart-Plange

Ghana Health Service

Estafanos Shargie

Global Fund

Research

Keywords: Malaria, mortality trends, Ghana

Posted Date: September 1st, 2020 
DOI: https://doi.org/10.21203/rs.3.rs-65589/v1

License: (a) (i) This work is licensed under a Creative Commons Attribution 4.0 International License. Read Full License 


\section{Abstract}

Background Multiple interventions have been implemented over the years to decrease malaria morbidity and mortality in Ghana. After years of rolling out these interventions, assessing its effect on the trends for decision making is key. The objective of this study was to understand the trends of malaria related deaths in the country from 2005 to 2014.

Methods Between September 2016 and June 2017, abstraction of retrospective data covering January 1st 2005 to December 31st, 2014 was conducted in 93 sampled health facilities providing AIDs/HIV, Tuberculosis and Malaria (ATM) services in Ghana. This paper is written out of a bigger study which looked at the mortality of ATM in Ghana. Abstraction form was used to retrieve socio-demographic and admission outcome of patients from facility registers, death certificates and inpatients registers. Data was entered using EpiData 3.1 statistical software package then exported to STATA 11 version for analysis. Bivariate analysis with Chi-square test and multiple logistic regression were done to assess factors associated with malaria related mortality at a $5 \%$ level of significance.

Results A total of 667,186 admissions records related to malaria were retrieved in 93 hospitals from 2005 to 2014 with majority of the admissions being females (53.9\%) and children under 5 years $(47.8 \%)$. A total of $10,433(1.6 \%)$ of the admitted malaria cases were reported to have died with males and children under five years accounting for $51.0 \%$ and $26.6 \%$ respectively. Malaria case fatality rate showed an increasing trend from $2.2 \%$ in 2005 to $3.0 \%$ in 2007 and decreased to $1.1 \%$ in 2014 . Malaria mortality declined by approximately $59 \%$ over the ten-year period with an average annual decline of $7 \%$. Year of admissions, age, sex, insurance status and ownership of facility was significantly associated with mortality $(p<0.001)$. Sub-regional level hospitals have a decreased likelihood of malaria related mortality. A non-insured client increases the likelihood of mortality by 2.4 times (Odds Ratio $=2.4 p<0.001$ ).

Conclusion Malaria mortality declined over the ten-year period with an average annual decline of $7 \%$. An increase in age and not having health insurance among malaria-related admissions increases the likelihood of mortality.

\section{Background}

Malaria is endemic in Ghana with the entire population at risk with the most vulnerable being children under five years, pregnant women and the immuno-compromised. The World Health Organization (WHO) estimated about 198 million cases of malaria and 584000 deaths globally in 2013. Malaria mortality rates have fallen by $47 \%$ and $54 \%$ globally and in Africa respectively since 2000 [1]. Most of the malaria deaths occurred among children living in Africa where a child dies every minute from malaria. Malaria mortality rates among children in Africa by 2013 have been reduced by an estimated 58\% since 2000[1]. Data from the District Health Information Management System for Ghana (DHIMS-II) 2014, revealed that malaria was a major cause of hospital attendance, comprising about $30 \%$ of Out-Patient Department 
(OPD) visits, $27.9 \%$ in-patients and $7.2 \%$ deaths [2]. The total number of deaths attributable to malaria in 2014 was 2,200 out of which 1,060 (48.2\%) occurred among children under 5 years [2].

There has been the roll out of several interventions in the country throughout the years to ensure reduction in malaria mortality and morbidity. These interventions such as the insecticide treated nets, seasonal malaria chemoprevention, intermittent preventive treatment in pregnant women are aimed at preventing the occurrence of the malaria disease. Strategies such as test, treat and track at the health facility and community levels also help diagnose and treat malaria cases appropriately. After several years of carrying out these interventions, it is important to assess the trends of malaria related morbidity and mortality in the context of increasing interventions. The objective of this paper is to describe the trends in the malaria-related deaths in the country from 2005 to 2014.

\section{Methods}

\section{Study design}

This study was done as part of a bigger one which looked at the mortality of AIDS/HIV, TB and malaria. A retrospective data analysis from a representative sample of health facilities providing services for the three diseases (ATM) was conducted.

\section{Study Site}

The study was conducted in all ten regions across the country. The study included teaching hospitals, regional hospitals, district hospitals and other primary level hospitals that provide inpatient services for AIDS/HIV, TB and Malaria (ATM).

\section{Study Period}

Data was collected from September 2016 to June 2017.

\section{Sample size and sampling procedure}

For a facility to be eligible for inclusion in the sampling frame, the study used the following eligibility criteria:

A facility should provide diagnosis and treatment as well as inpatient services for at least one year during the study period for the three diseases: AIDS/HIV, TB and Malaria. Out of the total number of health facilities in the country, 198 health facilities provided diagnosis and treatment services for HIV/AIDS, TB, and Malaria of which 169 facilities provided additional in-patient services. These 169 facilities were used as the sampling frame. Of the total 169 health facilities which qualified the study criteria (provided admission for ATM) 100 (59.2\%) were selected for the study. Facilities were stratified into regions and the number of health facilities in each region was allocated based on the total number of health facilities in that region. 
In each region, all teaching and regional hospitals were purposively sampled and the remaining facilities were selected randomly using excel generated random numbers proportionate to the number of ART sites as shown in Table 1 (With all region having a minimum of $50 \%$ of the eligible.

Table 1: Distribution of eligible health facilities and selected health facilities for ATM mortality study

\begin{tabular}{|llll|}
\hline Region & $\begin{array}{l}\text { Total eligible } \\
\text { facilities }\end{array}$ & $\begin{array}{l}\text { Number of facilities } \\
\text { selected }\end{array}$ & $\begin{array}{l}\text { Number of Facilities } \\
\text { assessed }\end{array}$ \\
\hline Ashanti & 29 & 16 & 16 \\
\hline Brong-Ahafo & 19 & 11 & 10 \\
\hline $\begin{array}{l}\text { Central } \\
\text { Region }\end{array}$ & 11 & 7 & 6 \\
\hline $\begin{array}{l}\text { Eastern } \\
\text { Region }\end{array}$ & 20 & 12 & 11 \\
\hline Greater Accra & 22 & 13 & 12 \\
\hline Northern & 15 & 9 & 8 \\
\hline Volta & 20 & 12 & 12 \\
\hline Western & 20 & 12 & 11 \\
\hline Upper East & 7 & 4 & 3 \\
\hline Upper West & 6 & 4 & 4 \\
\hline Total & 169 & 100 & 93 \\
\hline
\end{tabular}

\section{Data collection procedure}

A data abstraction form was used to retrieve data from facility registers, death certificates, inpatients registers and records covering the period January $1^{\text {st }} 2005$ to December $31^{\text {st }}, 2014$.

Extraction of data from the registers and various data forms were carried out by trained Research Assistants.

\section{Data entry and analysis}

Data was entered using a predesigned database format using EpiData 3.1 statistical software package and then exported to Stata (v11, Stata Corporation, USA) and SPSS (v20, IBM Corporation, USA) for analysis. Frequencies, proportions, and ratios were calculated to describe basic characteristics of study 
subjects. Trends in malaria-related mortality were determined. Bivariate analysis with Chi-square test was done to assess factors associated with ATM related mortality. A p-value of less than $5 \%$ was considered statistically significant. In order to explore the relative influence of each independent variable or factor on mortality and to test significance of association, we conducted a binary logistic regression analysis using a multivariate model.

Meta-analysis was used to evaluate the trends in death rates among inpatients over time and to have a pooled estimate of death rate. Death rate in malaria-related admission for each year was determined at $95 \% \mathrm{Cl}$. The point estimate of death rate, upper and lower value of the $95 \% \mathrm{Cl}$ were fed to the Metaanalysis syntax command. Weighting using random effect was applied since we expected heterogeneity across cohorts.

\section{Limitations of the study}

It did not fully assess risk factors for disease specific mortality because of limited data found in the records on variables.

\section{Results}

From the total 100 facilities selected for this study, we able to collect data from 93 (93\%) of facilities. A total of 667,186 admissions records related to malaria were retrieved in 93 hospitals from 2005 to 2014, with majority of the admissions [359,311(53.9\%)] being females. Admissions were highest in children under 5 years (47.8\%) followed by children between the ages 5 to 14 years old $(15.6 \%)$ whiles patients aged 65 to 74 years recorded the lowest (2.5\%). During the period under review, $19,779(3 \%)$ of the admissions were in pregnant women.

Northern region recorded the highest number of malaria admissions constituting $17.9 \%$ followed by Volta region (15.5\%) with Upper East region recording the lowest malaria admissions (1.3\%). Malaria admissions increased from $3.6 \%$ in 2005 to $17.8 \%$ in 2014 with district hospitals recording the highest (79.7\%) and Teaching Hospitals, the lowest (3.4\%). Among all the malaria cases abstracted, 411,732(61.7\%) were insured either on NHIS or Private Health Insurance Scheme. Among the admitted malaria cases, $85.4 \%$ had malaria as the principal diagnosis with $14.2 \%$ having malaria as additional diagnosis to other principal diagnosis. About $93.0 \%$ of all the recorded malaria cases admitted were discharged home. Of the total malaria related admissions, $10,433(1.6 \%)$ of them were reported dead with males accounting for $51.0 \%$; the highest proportion was recorded in children under five years $2,771(26.6 \%)$ whiles the lowest was recorded in patients aged 75years and above (12.0\%). Malaria deaths among admissions (case fatality rate) increased from $2.2 \%$ in 2005 to $3.0 \%$ in 2007 and declined from $3.0 \%$ in 2007 to $1.1 \%$ in 2014(Figure 1). Malaria mortality declined by approximately $59 \%$ over the ten-year period with an average annual decline of $7 \%$.

The highest case fatality rate (3.0\%) was recorded in Central region with Ashanti region having recorded the lowest (1.0\%). Even though children under five accounted the highest number of malaria mortalities 
$(2,771)$, that age group together with those aged 5-14years recorded the lowest $(0.9 \%)$ malaria case fatality rate while patients above 74 years recorded the highest CFR (7. 4\%). The Regional Hospitals recorded the highest case fatality rate (3.0\%). Case fatality rate was lower for insured patients $(1.1 \%)$ compared with non-insured patients $(2.9 \%)$.

Table 2: Factors Associated with Malaria Mortality in Ghana from 2005 to 2014 


\begin{tabular}{|c|c|c|c|c|}
\hline \multirow[t]{2}{*}{ Independent variables } & \multicolumn{4}{|c|}{ Patient died on Admission } \\
\hline & Yes, \# (\%) & No, \# (\%) & Total & $P$ value \\
\hline Year of admission & & & & 0.000 \\
\hline 2005 & $510(2.2)$ & 22391(97.8) & 22901 & \\
\hline 2006 & $626(2.6)$ & 23054(97.4) & 23680 & \\
\hline 2007 & 836(3.0) & 26961(97.0) & 27797 & \\
\hline 2008 & $909(2.3)$ & $38527(97.7)$ & 39436 & \\
\hline 2009 & $1010(2.0)$ & 49804(98.0) & 50814 & \\
\hline 2010 & 1295(1.9) & 67509(98.1) & 68804 & \\
\hline 2011 & $1275(1.6)$ & 77210(98.4) & 78485 & \\
\hline 2012 & $1403(1.4)$ & 99663(98.6) & 101066 & \\
\hline 2013 & $1362(1.3)$ & 107332(98.7) & 108694 & \\
\hline 2014 & $1207(1.1)$ & $112605(98.9)$ & 113812 & \\
\hline Total & 10433(1.6) & $625056(98.4)$ & 635489 & \\
\hline \multicolumn{5}{|l|}{ Sex of Patient } \\
\hline Male & $5317(1.8)$ & $287470(98.2)$ & 292787 & 0.000 \\
\hline Female & $5088(1.5)$ & $335689(98.5)$ & 340777 & \\
\hline No Information & $28(1.5)$ & 1897(98.5) & 1925 & \\
\hline Age group & & & & 0.000 \\
\hline$<5$ & 2771(.9) & $301417(99.1)$ & 304188 & \\
\hline $5-14$ & $858(.9)$ & 98368(99.1) & 99226 & \\
\hline $15-24$ & $642(1.0)$ & 66329(99.0) & 66971 & \\
\hline $25-34$ & $949(1.8)$ & $52723(98.2)$ & 53672 & \\
\hline $35-44$ & 1132(3.3) & $33423(96.7)$ & 34555 & \\
\hline $45-54$ & $1012(4.1)$ & 23763(95.9) & 24775 & \\
\hline $55-64$ & $831(5.2)$ & 15282(94.8) & 16113 & \\
\hline $65-74$ & $915(5.8)$ & 14861(94.2) & 15776 & \\
\hline $75+$ & 1249(7.6) & 15182(92.4) & 16431 & \\
\hline Regions & & & & 0.00 \\
\hline
\end{tabular}




\begin{tabular}{|lllll|}
\hline Greater Accra & $968(2.1)$ & $44250(98.9)$ & 45218 & \\
\hline Ashanti & $788(1.0)$ & $81968(99.0)$ & 82756 & \\
\hline Brong Ahafo & $815(1.2)$ & $68591(98.8)$ & 69406 & \\
\hline Eastern & $1490(1.9)$ & $78484(98.1)$ & 79974 & \\
\hline Northern & $1072(0.9)$ & $112360(99.1)$ & 113432 & \\
\hline Upper East & $77(0.9)$ & $8758(99.1)$ & 8835 & \\
\hline Upper West & $558(2.2)$ & $25212(97.8)$ & 25770 & \\
\hline Western & $963(1.7)$ & $54254(98.3)$ & 55217 & \\
\hline Volta & $2102(2.1)$ & $99294(97.9)$ & 101396 & \\
\hline Central & $1600(3.0)$ & $51885(97.0)$ & 53485 & \\
\hline Type of facility & & & & \\
\hline Teaching Hospital & $502(2.3)$ & $21792(97.7)$ & 22294 & \\
\hline Regional Hospital & $1567(3.0)$ & $50722(97.0)$ & 52289 & \\
\hline District Hospital & $7807(1.5)$ & $503009(98.5)$ & 510816 & \\
\hline Other Hospital & $557(1.1)$ & $49533(98.9)$ & 50090 & \\
\hline Facility Ownership & & & & \\
\hline Government & $8256(1.9)$ & $435412(98.1)$ & 443668 & \\
\hline Quasi-Government & $329(1.0)$ & $31262(99.0)$ & 31591 & \\
\hline Private & $26(0.5)$ & $5077(99.5)$ & 5103 & \\
\hline Faith-based & $1822(1.2)$ & $153305(98.8)$ & 155127 & \\
\hline Health insurance Status & & & & \\
\hline Yes & $4435(1.1)$ & $391511(98.9)$ & 395946 & \\
\hline No & $3552(2.9)$ & $117287(97.1)$ & 120839 & \\
\hline No Information & $2446(2.1)$ & $116258(97.9)$ & 118704 & \\
\hline
\end{tabular}

Year of admissions, age, sex, insurance status, type and ownership of facility were significantly associated with mortality $(p<0.001)$ (Table 2). The regional hospitals have about $24 \%$ (Odds Ratio=1.24, $\mathrm{p}-<0.001)$ increased odds of mortality compared to teaching hospitals. Cases of malaria admitted into Private, Faith-based and Quasi-government facilities had a decreased likelihood of dying compared to government facilities (Table 3). A unit increase in age among malaria related admissions had a 0.3 increased odds of mortality (Odds Ratio $=1.029, p<0.001$ ). 
Table 3: Multivariate analysis of factors associated with mortality among malaria admissions, Ghana from 2005 to 2014. 


\begin{tabular}{|c|c|c|c|c|c|}
\hline \multirow[t]{2}{*}{ Variable } & \multirow[t]{2}{*}{ B } & \multirow[t]{2}{*}{ Sig. } & \multirow[t]{2}{*}{$\operatorname{Exp}(B)$} & \multicolumn{2}{|c|}{ 95\% C.I. } \\
\hline & & & & Lower & Upper \\
\hline Age & .028 & 0.000 & 1.029 & 1.028 & 1.029 \\
\hline Male & & .000 & & & \\
\hline Female & -.447 & .000 & .639 & .614 & .665 \\
\hline No Information & -.220 & .355 & .802 & .503 & 1.280 \\
\hline 2005 & & .000 & & & \\
\hline 2006 & .197 & .001 & 1.217 & 1.079 & 1.373 \\
\hline 2007 & .243 & .000 & 1.275 & 1.137 & 1.431 \\
\hline 2008 & .192 & .001 & 1.212 & 1.082 & 1.357 \\
\hline 2009 & .102 & .071 & 1.108 & .991 & 1.238 \\
\hline 2010 & .117 & .033 & 1.124 & 1.009 & 1.251 \\
\hline 2011 & -.034 & .541 & .967 & .868 & 1.077 \\
\hline 2012 & -.180 & .001 & .836 & .750 & .931 \\
\hline 2013 & -.251 & .000 & .778 & .698 & .867 \\
\hline 2014 & -.362 & .000 & .696 & .624 & .777 \\
\hline Teaching Hospital & & .000 & & & \\
\hline Regional Hospital & .211 & .000 & 1.235 & 1.111 & 1.373 \\
\hline District Hospital & -.429 & .000 & .651 & .592 & .717 \\
\hline Other Hospital & -.868 & .000 & .420 & .358 & .492 \\
\hline Government & & .000 & & & \\
\hline Quasi-Government & -.474 & .000 & .622 & .532 & .728 \\
\hline Private & -1.067 & .000 & .344 & .225 & .526 \\
\hline Faith-based & -.273 & .000 & .761 & .719 & .806 \\
\hline Health Insurance & & 0.000 & & & \\
\hline No Health Insurance & .893 & .000 & 2.442 & 2.328 & 2.561 \\
\hline No Information & .696 & .000 & 2.005 & 1.897 & 2.119 \\
\hline HIV/AIDS & & 0.000 & & & \\
\hline TB & -.500 & .001 & .607 & .449 & .820 \\
\hline
\end{tabular}




\begin{tabular}{|lrrrrr|} 
Malaria & -2.178 & .000 & .113 & .098 & .131 \\
Other & -1.484 & .000 & .227 & .196 & .262 \\
No Information & -1.233 & .001 & .291 & .145 & .584 \\
Constant & -2.409 & .000 & .090 & & \\
Model R2=0.018, X2=11469.710, df=24, P=0.000 & \\
\hline
\end{tabular}

Among malaria-related admissions, Patients with malaria as principal diagnosis have decreased odds $(\mathrm{OR}=0.11, \mathrm{P}<0.001)$ of dying compared to HIV/AIDS as principal diagnosis. All other disease as principal diagnosis with HIV/AIDS as reference also had decreased likelihood. Females have a significant $36 \%$ decreased odds of malaria mortality (Odds Ratio $=0.64, p<0.001)$ compared to males and being a noninsured client increased the likelihood of mortality by 2.4 times (Odds Ratio=2.4 $p<0.001$ ). (Table 3 ).

\section{Discussion}

Assessing trends of malaria morbidity and mortality is one of the important tools in measuring the impact of interventions over the years and data capturing gaps. Over the years, the National Malaria Control Programme has implemented multiple interventions with the help of Global Fund to decrease the malaria morbidity and mortality in Ghana. This is manifested in survey findings which shows that households who owned at least one ITN increased from $18.7 \%$ in 2003[3], to $73 \%$ in 2016[4]. Healthseeking behaviour has improved over the years with advice or treatment sought for $49.4 \%$ of children with fever in the 2 weeks before the survey in 2011[5] increasing to $72 \%$ in 2016[4].

In this study, mortality among malaria admissions (Case Fatality Rate) showed an upward trend from $2.2 \%$ in 2005 to $3.0 \%$ in 2007 and a downward trend from $3.0 \%$ in 2007 to $1.1 \%$ in 2014 . National Health Insurance Scheme (NHIS) in Ghana was introduced in 2003 which made health care more accessible to people thereby increasing the number of people who sought care and were admitted. Though Artemisinin Combination Therapies (ACTs) were introduced in 2005, coverage was low and increased over time. It was in 2007 that the impact of all the interventions especially the use of ACTs started manifesting. This coupled with increased number of NHIS clients and increasing sub-district facilities across the country may have contributed to decreasing delays in care seeking by patients and a further decrease in presenting with very fatal forms of the disease. The trend could also be due to improved quality of malaria case management by health workers as case management trainings have increased over the years $[6,7]$ with increase in country donor support. The cumulative effect may have started manifesting after the year 2007 as seen in other researches [8-10]. 
Teaching and Regional hospitals have increased likelihood for malaria-related mortality compared to the lower facilities. This could be resulting from the fact that regional and teaching hospitals are referral levels where complicated cases (with high risk of dying) are sent to be managed. Malaria-related admissions in government facilities had an increase likelihood of dying compared to the other types of ownership. Though the private sector and some public sector (district and sub-district hospitals) health facilities are known to be the first point of call for cases especially malaria [11-13], cases tend to be referred to the higher facilities (Regional and Teaching Hospitals) which are often public. It is worthy of note that all the regional and teaching hospitals are public/government facility. The findings may have therefore have resulted from improved quality of care in the non-governmental facilities or admission of relatively non-severe cases in the lower and private facilities compared to the regional facilities. This increases the likelihood of the government facilities having potentially fatal cases.

In the bivariate analysis, region of admission was significantly associated with mortality. The Central Region recording the highest CFR (3.0\%) while Upper East and Northern Regions recorded the lowest $(0.9 \%)$. This could be due to varying case management challenges across the regions, late reporting of cases and uneven distribution of capacity to manage such cases across the country. This is on the backdrop that Central Region is not the region with the worst Doctor: Patient ratio [14] and not the poorest in Ghana [15] which are variables that could worsen CFR rate yet the region recorded the highest CFR.

Increase in age has a significant $0.2 \%$ increased likelihood of malaria-related mortality. This could have resulted from the high attention given to the management of fevers in children over the years and possible delays in seeking health care by adults as compared to taking their children to the health facility when the need arose. The theory of older ages becoming more vulnerable to malaria than before due to a gradual loss of partial immunity as malaria burden decreased following intensified malaria control measures could also be the reason. This has been documented in some researches [16-18].

Among malaria-related admissions, patients with malaria as principal diagnosis have decreased odds $(\mathrm{OR}=0.11, \mathrm{P}<0.001)$ of dying compared to having HIV/AIDS as principal diagnosis. Having any other disease as principal diagnosis using HIV/AIDS as reference also showed a decreased likelihood of dying. This may have resulted because patients admitted with HIV/AIDS as principal diagnosis may be severely ill and in an immune-compromised state which may get complicated with other diseases therefore increasing the odds of dying.

Finally, not having health insurance increases the likelihood of malaria-related mortality by 2.34 times. This could be linked to the fact that insurance holders are more likely to seek health care early and in an appropriate place and are therefore less likely to present with severe forms of the disease. They are also likely to be provided with all the resources for their management especially if there is the need for management of concomitant diseases. This health insurance finding is supported by similar trends seen in some researches $[19,20]$.

\section{Conclusion}


Declines in malaria cases and deaths were higher in children under five years old than in all age groups. Malaria mortality declined by approximately $59 \%$ over the ten-year period with an average annual decline of $7 \%$. The likelihood of dying after malaria-related diagnosis in regional hospitals and government hospitals was higher than in the other type and ownership of facilities respectively. An increase in age and not having health insurance among malaria-related admissions increases the likelihood of mortality. Targeted interventions among elderly and an intervention to increase health insurance coverage may help the malaria control program to reduce malaria-related mortality in the country.

\section{List Of Abbreviations}

\begin{tabular}{ll} 
ATM & AIDS, Tuberculosis and Malaria \\
\hline DHMIS & District Health Management Information System \\
\hline ERC & Ethical Review Committee \\
\hline GFATM & Global Fund to Fight AIDS Tuberculosis and Malaria \\
\hline GHS & Ghana Health Service \\
\hline NMCP & National Malaria Control Programme \\
\hline OPD & Out Patient Department \\
\hline WHO & World Health Organization
\end{tabular}

\section{Declarations}

\section{Ethics approval and consent to participate}

Ethical approval was obtained from the Ghana Health Service Ethical Review Committee (ERC) with approval number GHS-ERC 12/05/16. Permission was sought from all the Regional Health Directorates, Chief Executive Officers of the various Teaching Hospitals, Medical Directors and Medical Superintendents of all the selected health facilities included in the study. All the information obtained from this study was kept confidential.

\section{Consent for publication}

Not applicable

\section{Availability of data and materials}


Data used in this study is available and will be provided by the lead author upon request.

\section{Competing interests}

The authors declare that they have no competing interests.

\section{Authors' contributions}

KLM, FAB, SAA, MS, DL, ES designed the study. KLM participated in the data collection, statistical analysis and drafted the manuscript. NYP, NAB, WH, MJ ZA participated in data collection, statistical analysis and reviewed the manuscript for intellectual content. CBP reviewed the manuscript for intellectual content. All authors read and approved the final version of the manuscript.

\section{Acknowledgements}

On behalf of the Ghana Health Service and myself, I wish to express my profound gratitude to all who made this study possible. Special appreciation goes to the leadership of the GHS at the Regional and District Health Directorates as well as health facilities for their immense support during the study period. The regional and facility Health Information Officers deserve commendation for their coordination and supervisory role for this important project. All Teaching Hospitals who participated in the study played a significant role and we are most grateful. This project would not have been successful without the active role of the data collectors in data collection, entry and transmission.

\section{References}

1. World Health Organization. 2103 World Malaria Report; 2014.

2. National Malaria Control. 2014 Annual Report National Malaria Control [Internet]. Accra; 2014. Available from: http://www.ghanahealthservice.org/downloads/NMCP_2014_ANNUAL_REPORT.pdf.

3. Ghana Statistical Service. 2003 Demographic and Health Survey report; 2004.

4. Ghana Statistical Service. 2016 Malaria Indicator Survey report; 2017.

5. Ghana Statistical Service. 2011 Ghana Multiple Indicator Cluster Survey [FR262] - FR262.pdf. 2012.

6. Mahaba Hisham M. Impact of Training of Primary Health Care Staff on Maternity and Child Health Services https://www.ncbi.nlm.nih.gov/pmc/articles/PMC3437164/.

7. The Impact of Training Program on Organizational Effectiveness: A Case Study of Healthcare Organization in Karachi 
Zulfiqar A, Mehwish H, The Impact of Training Program on Organizational Effectiveness: A Case Study of Healthcare Organization in Karachi-Pakistan. https://www.omicsonline.org/openaccess/the-impact-training-program-organizational-effectiveness-case-study-of-healthcareorganization-karachipakistan-2167-0331.1000120.php.

8. Aregawi MW, Ali AS, Al-mafazy A, Molteni F, Katikiti S, Warsame M, et al. Reductions in malaria and anaemia case and death burden at hospitals following scale-up of malaria control in Zanzibar, 1999-2008. Malar J. 2011;10:46.

9. Ceesay SJ, Casals-Pascual C, Erskine J, Anya SE, Duah NO, Fulford AJC, et al. Changes in malaria indices between 1999 and 2007 in The Gambia: a retrospective analysis. Lancet. 2008 Nov;372(9649):1545-54.

10. Karema C, Aregawi MW, Rukundo A, Kabayiza A, Mulindahabi M, Fall IS, et al. Trends in malaria cases, hospital admissions and deaths following scale-up of anti-malarial interventions, 2000-2010, Rwanda. Malar J. 2012;11:236.

11. Akande TM. Referral system in Nigeria: Study of a tertiary health facility. Ann Afr Med. 2004;3:130-3.

12. Nkyekyer Kobinah. Peripartum referrals to Korle Bu Teaching Hospital, Ghana - a descriptive study.

13. 13. Ministry of Health, Ghana Referral Policy and Guidelines. 2012.

14. Ghana Health. Service the health sector in Ghana, Facts and figures, 2017 ghanahealthservice.org/downloads/Facts + Figures_2018.pdf.

15. Ghana Statistical Service. Ghana Living Standard Survey (GLSS 7) 2017, http://www.statsghana.gov.gh/nada/index.php/catalog/97/study-description.

16. Griffin JT1. Ferguson NM1 „, Ghani AC1. Estimates of the changing age-burden of Plasmodium falciparum malaria disease in sub-Saharan Africa. 2014.

17. Meghna Desai AM, Buff S, Khagayi P, Byass N, Amek A, van Eijk L, Slutsker J, Vulule FO, Odhiambo PA, Phillips-Howard KA, Lindblade, Kayla F, Laserson, Mary J. Hamel. Age-Specific Malaria Mortality Rates in the KEMRI/CDC Health and Demographic Surveillance System in Western Kenya, 20032010. 2014.

18. Snow RW1, Omumbo JA, Lowe B, Molyneux CS, Obiero JO, Palmer A, Weber MW, Pinder M, Nahlen B, Obonyo C, Newbold C, Gupta S, Marsh K. Relation between severe malaria morbidity in children and level of Plasmodium falciparum transmission in Africa. 1997.

19. Wielen $N$ van der, Channon AA, Falkingham J. Does insurance enrolment increase healthcare utilisation among rural-dwelling older adults? Evidence from the National Health Insurance Scheme in Ghana. BMJ Glob Heal. 2018 Feb;3(1):e000590.

20. Werner WJ. Micro-insurance in Bangladesh: Risk Protection for the Poor? J Health Popul Nutr. 2009 Aug;27(4):563-73.

\section{Figures}




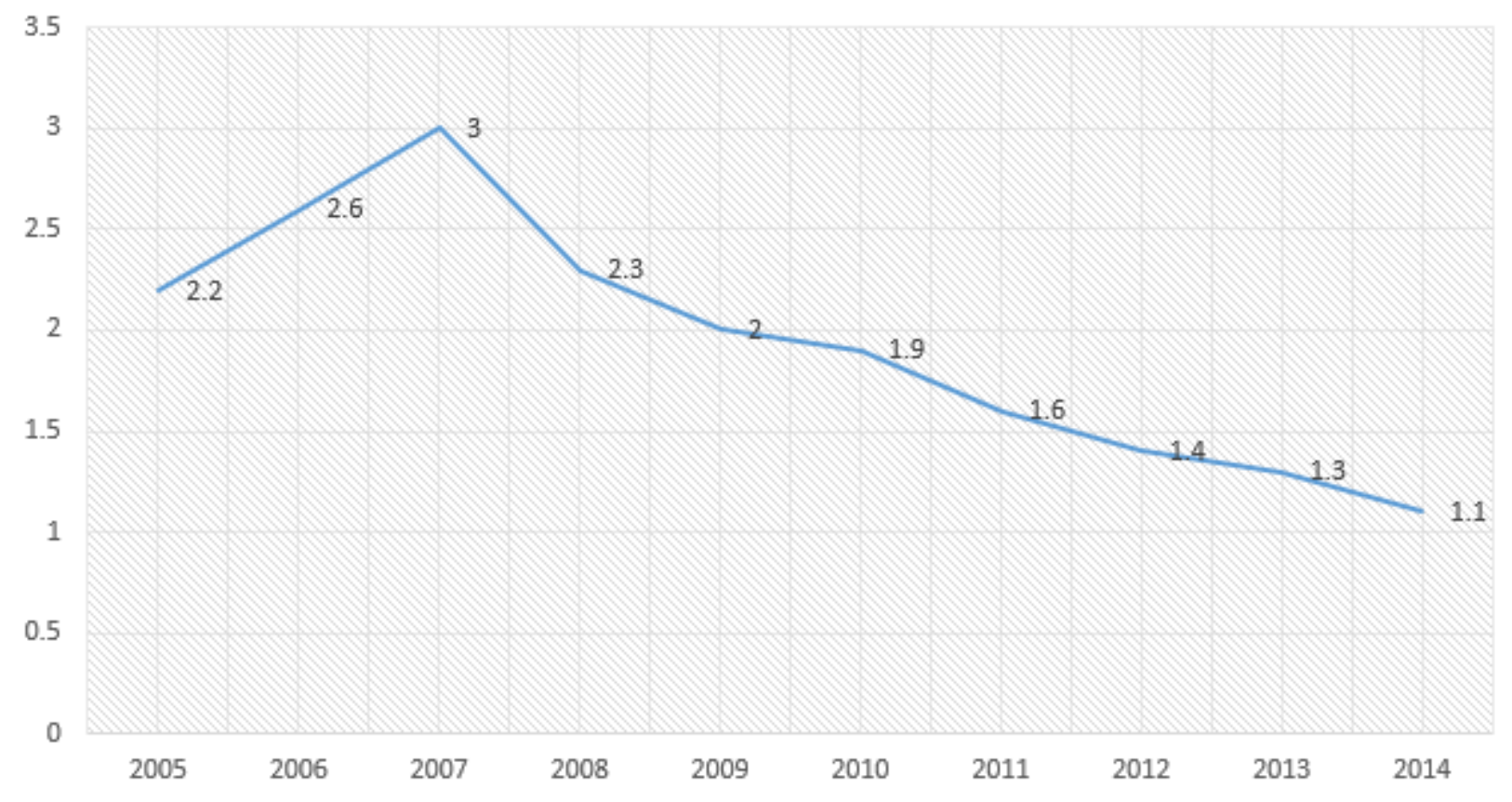

Figure 1

Trends of mortality rate among Malaria related admissions in Ghana from 2005 to 2014 